\title{
EFEITOS DA INCLUSÃO DE DIFERENTES NÍVEIS DO FARELO DE BABAÇU NA DIETA DE SUÍNOS
}

Marco Aurélio Lopes Della Flora ${ }^{1}$ Marcos Paulo Ferreira de Albuquerque ${ }^{1}$

Matheus Mendes ${ }^{1}$

Rayssa de Sousa Rego ${ }^{1}$

Renata Rodrigues Viana ${ }^{1}$

Wellington Matheus Cavalcante ${ }^{1}$

Welligton Conceição da Silva ${ }^{2}$

Éder Bruno Rebelo da Silva ${ }^{1}$

Raimundo Nonato Colares Camargo Junior ${ }^{1}$

\section{RESUMO}

O farelo de babaçu tem sido utilizado na agricultura familiar, apesar do desconhecimento de seu valor nutricional. Assim, torna-se necessária a realização de pesquisas para estabelecer padrões de utilização e análises laboratoriais para determinação de suas características nutricionais, a fim de se conhecer seus efeitos na nutrição e crescimento de suínos. Neste contexto, o objetivo neste estudo foi avaliar o valor nutricional e o desempenho zootécnico de suínos em crescimento, alimentados com diferentes níveis de inclusão do farelo de babaçu na dieta. $\mathrm{O}$ estudo foi conduzido na Unidade Integrada de Ensino, Pesquisa e Extensão (UNIEPE) de Suinocultura do Instituto Federal do Pará (IFPA) campus Marabá Rural. Foram utilizados 16 suínos mestiços com peso inicial de 7,63 $\pm 1,31 \mathrm{~kg}$, distribuídos aleatoriamente em quatro baias. $\mathrm{O}$ delineamento experimental foi inteiramente casualizado, com quatro tratamentos $(0 \%, 10 \%, 20 \%$ e $30 \%)$ em quatro repetições. Avaliou-se as variáveis ganho de peso e valor nutricional. Os dados foram submetidos à análise de variância e regressão polinomial, ao nível de $5 \%$ de probabilidade. Como resultados constatou-se que a composição nutricional do farelo de babaçu é rico em matéria seca $(89,08 \%)$ e fibra bruta $(46,84 \%)$. Além disso, o ganho de peso dos leitões foi influenciado pela inclusão do babaçu na dieta. Essa variável apresentou efeito linear negativo $(\mathrm{P}<0,05)$, com coeficiente de determinação $\left(\mathrm{r}^{2}\right) \mathrm{de}$ 0,88. Portanto, foi possível concluir que a adição do farelo de babaçu reduz de forma linear o crescimento de leitões com inclusão de até $30 \%$ na dieta.

Palavras chave: desempenho, subproduto, suinocultura.

\section{EFFECTS OF DIFFERENT LEVELS OF BABAÇU BRAN ON THE SWINE DIET}

\begin{abstract}
Babassu meal has been used in family farming, despite its lack of nutritional value. Furthermore, it is necessary to carry out research to establish usage patterns and laboratory tests to determine their nutritional characteristics, in order to know their effects on the nutrition and growth of their pigs. In this context, the objective of this study, was to evaluate the nutritional value and/or zootechnical performance of growing pigs, fed with different

\footnotetext{
${ }^{1}$ Docente do Instituto Federal de Educação, Ciência e Tecnologia do Pará-IFPA. marco.dellaflora@ifpa.edu.br

${ }^{2}$ Universidade Federal Rural da Amazônia - UFRA. *Correspondência: welligton.medvet@gmail.com
} 
levels of inclusion of babassu meal in the diet. The study was carried out at the Integrated Unit for Training, Research and Extension (UNIEPE) of the Swine Industry of the Federal Institute of Pará (IFPA) Rural campus of Marabá. Sixteen mixed pigs with an initial weight of $7.63 \pm 1.31 \mathrm{~kg}$ were used, randomly distributed in four pens. The experimental design was internally randomized, with four treatments $(0 \%, 10 \%, 20 \%$ and $30 \%)$ in four repetitions. Were evaluated the varying weight gain and nutritional value. Data are subjected to analysis of variance and polynomial regression at a 5\% probability level. As a result, it appears that the nutritional composition of babassu bran is rich in dry matter $(89.08 \%)$ and crude fiber (46.84\%). In addition, the weight gain, the piglets, was influenced by the inclusion of babassu in the diet. This variable has a negative linear effect $(\mathrm{P}<0.05)$, with a coefficient of determination $\left(\mathrm{r}^{2}\right)$ of 0.88 . Therefore, it is possible to conclude that the addition of babassu meal linearly reduced the growth of piglets with the inclusion of up to $30 \%$ of the diet.

Keywords: performance, by-product, pig farming.

\section{EFECTOS DE DIFERENTES NIVELES DE SALVADO DE BABAÇU EN LA DIETA PORCINA}

\section{RESUMEN}

El salvado de babasú se ha utilizado en la agricultura familiar, a pesar de su falta de valor nutricional. Además, es necesario realizar investigaciones para establecer patrones de uso y pruebas de laboratorio para determinar sus características nutricionales, con el fin de conocer sus efectos en la nutrición y crecimiento de sus cerdos. En este contexto el objetivo de este estudio, fue evaluar el valor nutricional y / o desempeño zootécnico de cerdos en crecimiento, alimentados con diferentes niveles de inclusión de harina de babasú en la dieta. El estudio se realizó en la Unidade Integrada de Ensino, Pesquisa e Extensão (UNIEPE) de la Industria Porcina del Instituto Federal de Pará (IFPA) Campus Rural de Marabá. Se utilizaron dieciséis cerdos mixtos con un peso inicial de 7,63 $\pm 1,31 \mathrm{~kg}$, distribuidos aleatoriamente en cuatro corrales. El diseño experimental fue aleatorizado internamente, con cuatro tratamientos $(0 \%$, $10 \%, 20 \%$ y $30 \%$ ) en cuatro repeticiones. Regístrese variando el aumento de peso y el valor nutricional. Los datos se someten a análisis de varianza y regresión polinomial a un nivel de probabilidad del 5\%. Como resultado, parece que la composición nutricional del salvado de babasú es rica en materia seca $(89,08 \%)$ y fibra cruda (46,84\%). Además, la ganancia de peso, dos lechones, se vio influida por la inclusión de babasú en la dieta. Esta variable tiene un efecto lineal negativo $(\mathrm{P}<0.05)$, con un coeficiente de determinación $\left(\mathrm{r}^{2}\right)$ de 0.88 . Por tanto, es posible concluir que la adición de salvado de babasú redujo linealmente el crecimiento de los lechones con la inclusión de hasta un $30 \%$ de la dieta.

Palabras clave: performance, subproducto, cría de cerdos.

\section{INTRODUÇÃO}

A produção comercial de suínos na região norte do Brasil ainda é insuficiente para atender a demanda da população em geral. Em 2020 foram abatidas cerca de 49.3 milhões de cabeças de suínos, apresentando um aumento de 6,4\% ao comparar com o ano de 2019 (1). 
Dentre alguns fatores que colaboram para esse cenário, a flutuabilidade nos preços de ingredientes convencionais como milho e soja, se destacam como limitante para o avanço da cadeia produtiva, resultando em aumento dos custos de produção e redução do retorno financeiro aos produtores locais (2).

O estado do Pará apresenta diversidade de relevo e clima, com ampla disponibilidade de alimentos alternativos de origem vegetal, como, por exemplo, os oriundos da palmeira do babaçu (Orbignya ssp.), também conhecida como guaguaçu, uauaçu, gebara-uçu, coco-demacaco, coco-de-palmeira, coco-naiá, dependendo da região do país $(3,4)$.

Essa palmeira atinge cerca de 20 metros de altura, frutifica a partir do sétimo ano, com pico de produção aos 15 anos e vida produtiva em média de 35 anos. Produz de três a seis cachos de frutos por ano com cerca de 150 a 300 cocos, sendo que cada um possui três amêndoas. Tem sua origem na Amazônia, ocorre em áreas de terra firme, caracterizada como monocaule, crescimento lento; possui base protegida por bainhas foliares persistentes. Apresenta frutos ovóides, com a extremidade pontiaguda e cálice persistente na base, de 9 a $10 \mathrm{~cm}$ de diâmetro longitudinal e 4 a $5 \mathrm{~cm}$ de diâmetro transversal, pesando $27 \mathrm{~g}$, em média, com casca fibroso-coriácea, cor marrom ferrugínea. É considerado uma extraordinária matéria prima para a produção de óleo. Nesse processamento obtém-se o farelo, assim como o mesocarpo, considerados ingredientes alternativos para alimentação animal (5).

O coco babaçu possui epicarpo (11\%), mesocarpo (23\%), endocarpo (59\%) (6) e amêndoas (7\%) (7), dos quais pode ser extraído óleos (alimentícios e industriais), carvão vegetal, álcool e gás combustível (8). Seu farelo é um coproduto da extração do óleo da amêndoa, rico em proteínas e carboidratos, com potencial para ser empregado na alimentação animal, enquanto que o mesocarpo a parte rica em açucares é oriundo do processo de separação do mesocarpo e epicarpo do coco por extração mecânica (9). Sua disponibilidade ocorre justamente no período de entressafra do milho, apresentando-se como uma alternativa a esse ingrediente para pequenos produtores regionais, reduzindo os custos de produção, diminuindo o êxodo rural e auxiliando na subsistência dessas famílias.

Esse alimento tem sido utilizado na agricultura familiar, empiricamente, apesar do desconhecimento de seu valor nutricional. É necessária a realização de pesquisas para estabelecer padrões de utilização e análises laboratoriais para determinação de suas características nutricionais, a fim de se conhecer seus efeitos na nutrição e crescimento de suínos, conforme realizado no estudo de Gomes et al. (10).

Baseado nessas informações, hipotetiza-se: que a adição do farelo de babaçu pode afetar a desempenho dos suinos. Assim, o objetivo neste estudo foi avaliar o valor nutricional e o desempenho zootécnico de suínos em crescimento, alimentados com diferentes níveis de inclusão do farelo de babaçu na dieta.

\section{MATERIAL E MÉTODOS}

\section{Local}

O estudo foi conduzido na Unidade Integrada de Ensino, Pesquisa e Extensão (UNIEPE) de Suinocultura do Instituto Federal do Pará (IFPA) campus Marabá Rural (05 21' 54" S e 049 07' 24" W) (Figura 1), durante período de 25 dias, respeitando o período de adaptação de 30 dias. 

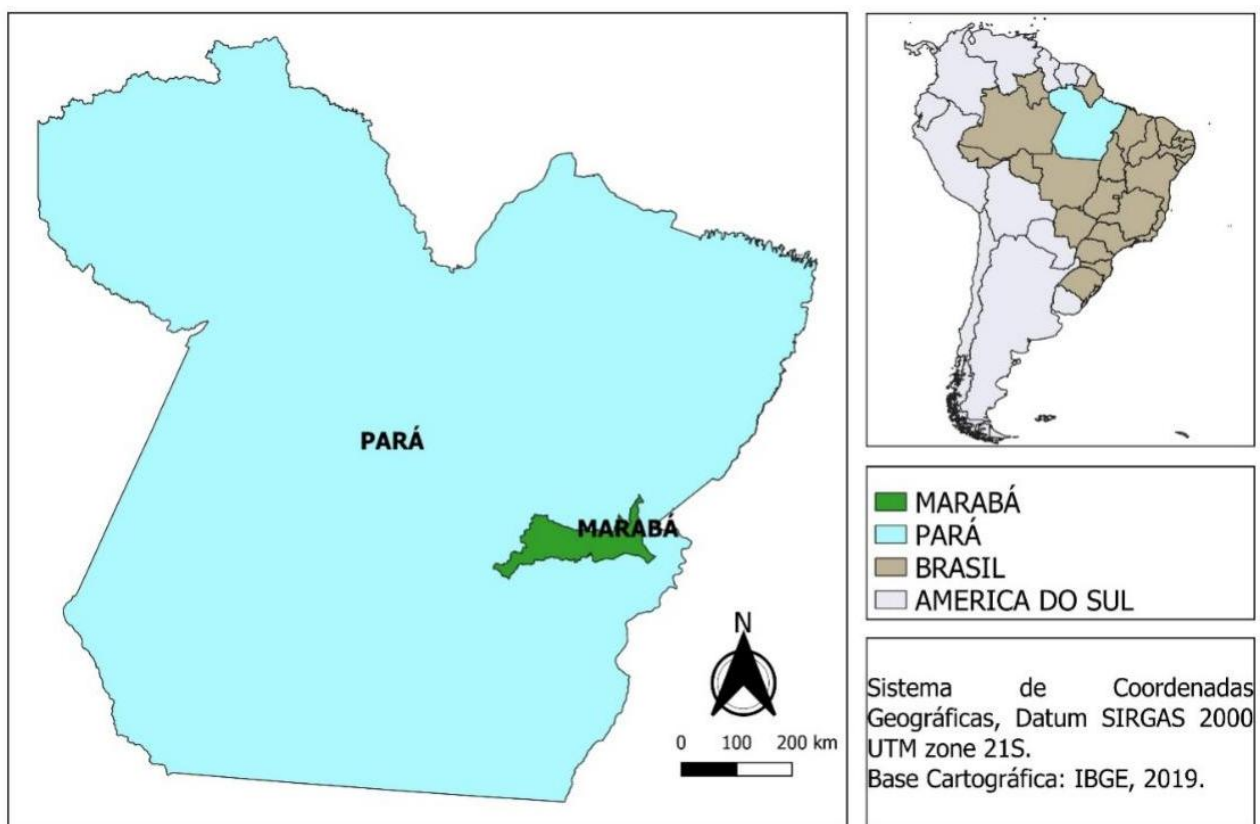

Figura 1. Mapa da localização do município do estudo.

\section{Animais experimentais}

Foram utilizados 16 suínos mestiços com peso inicial de 7,63 $\pm 1,31 \mathrm{~kg}$, oriundos da Instituição Educacional de Presidente Epitacio - UNIEPE. Foram distribuídos aleatoriamente em quatro baias contendo piso de concreto, dotadas de comedouros, bebedouros tipo chupeta, localizadas em prédio de alvenaria coberto com telhas de cerâmica.

\section{Delineamento experimental}

O delineamento experimental foi inteiramente casualizado, com quatro tratamentos:

$\mathrm{T}_{1}=$ nível de inclusão de farelo de babaçu na dieta $0 \%$;

$\mathrm{T}_{2}=$ nível de inclusão de farelo de babaçu na dieta $10 \%$;

$\mathrm{T}_{3}=$ nível de inclusão de farelo de babaçu na dieta $20 \%$; e

$\mathrm{T}_{4}=$ nível de inclusão de farelo de babaçu na dieta $30 \%$;

Foi considerado quatro repetições, visto que cada animal corresponde a uma unidade experimental (Tabela 1). O experimento foi conduzido após o período de desmame em um total de 25 dias.

Tabela 1. Formulação das dietas experimentais.

\begin{tabular}{ccccc}
\hline Ingredientes & \multicolumn{4}{c}{ Níveis de inclusão na dieta } \\
\cline { 2 - 5 } & $0 \%$ & $10 \%$ & $20 \%$ & $30 \%$ \\
\hline Soja & 20,00 & 20,00 & 20,00 & 20,00 \\
Milho & 75,00 & 65,00 & 55,00 & 45,00 \\
Babaçu & 0,00 & 10,00 & 20,00 & 30,00 \\
Núcleo & 5,00 & 5,00 & 5,00 & 5,00 \\
\hline
\end{tabular}

Della Flora MAL, Albuquerque MPF, Mendes M, Rego RS, Viana RR, Cavalcante WM et al. Efeitos da inclusão de diferentes níveis do farelo de babaçu na dieta de suínos. Vet. e Zootec. 2021; v28: 001-008. 


\section{Dieta}

A dieta referência $(0 \%$ de inclusão) foi formulada à base de milho, farelo de soja e núcleo para crescimento, de acordo com as exigências nutricionais para essa fase, seguindo-se as recomendações de Rostagno et al. (11). Os animais tiveram água disponível à vontade e a dieta foi fornecida duas vezes ao dia, 8:00 e 17:00h.

\section{Análise Bromatológica}

A análise bromatológica, constituída de matéria seca, cinzas, extrato etéreo, proteína bruta, fibra bruta e energia bruta do ingrediente teste, foi realizada seguindo recomendações da AOAC (12).

O ganho de peso dos animais foi calculado de acordo com a equação:

Na qual:

$$
G P=[P F-P I]
$$

$\mathrm{GP}=$ ganho de peso;

$\mathrm{PF}=$ peso final; $\mathrm{e}$

$\mathrm{PI}=$ peso inicial.

\section{Análise estatística}

Os dados foram organizados em planilhas do Microsoft Office ${ }^{\circledR}$ Excel 2014. Posteriormente, estes foram submetidos à análise de variância e regressão polinomial, ao nível de 5\% de probabilidade com o auxílio do programa estatístico SPSS 13.0.

\section{RESULTADOS}

A composição nutricional do farelo de babaçu pode ser observada na Tabela 2. Nota-se que esse subproduto é rico em matéria seca $(89,08 \%)$ e fibra bruta $(46,84 \%)$.

Tabela 2. Caracterização bromatológica do farelo de babaçu.

\begin{tabular}{cc}
\hline Nutriente & Farelo de babaçu \\
\hline Matéria seca (\%) & 89,08 \\
Proteína bruta (\%) & 2,80 \\
Extrato etéreo (\%) & 4,89 \\
Energia bruta (cal/g) & 4.266 \\
Fibra bruta (\%) & 46,84 \\
Cinzas (\%) & 1,86 \\
\hline
\end{tabular}

O Ganho de peso dos leitões foi influenciado pela inclusão do babaçu na dieta (Figura 2). Essa variável apresentou efeito linear negativo $(\mathrm{P}<0,05)$, com coeficiente de determinação $\left(\mathrm{r}^{2}\right)$ de 0,88 . Por ter sido prensado com casca, apresentou elevados teores de fibra bruta, sendo esse um dos principais fatores para a redução do ganho de peso dos animais. 


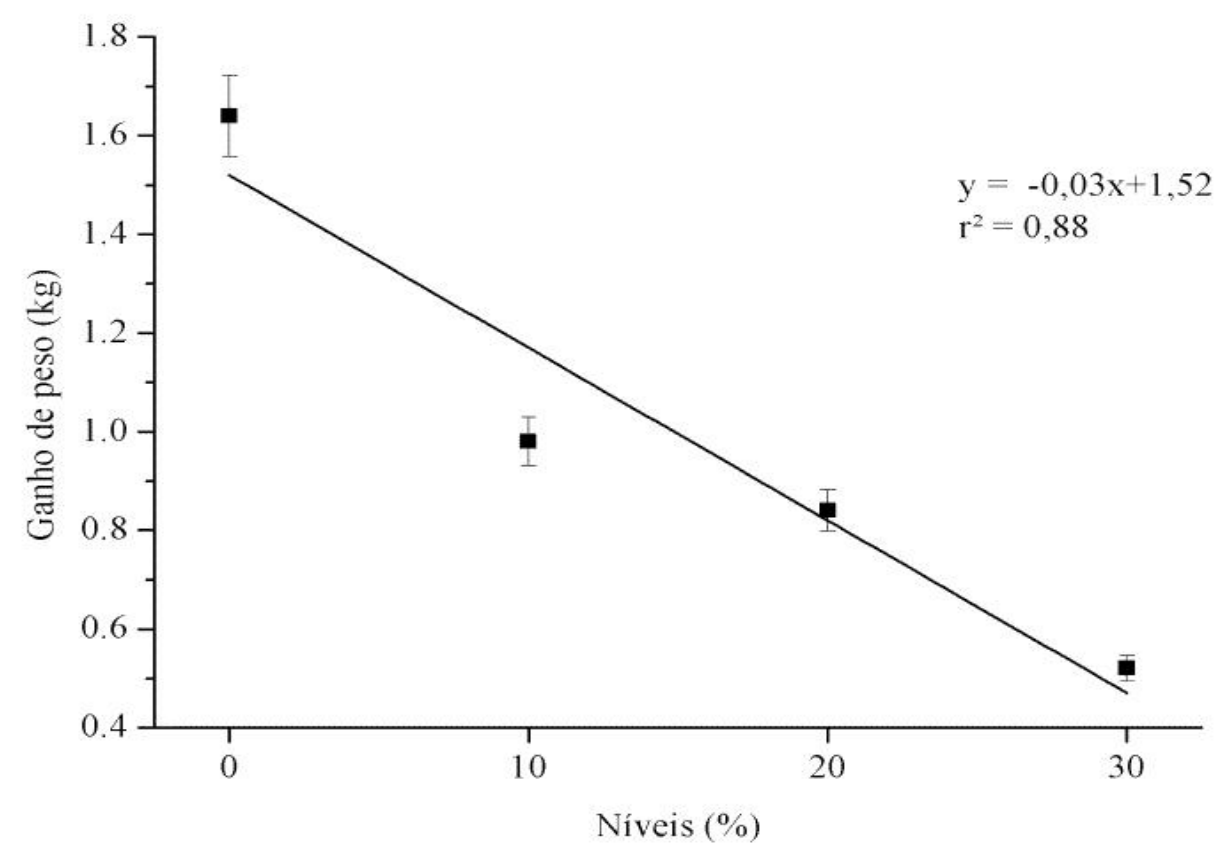

Figura 2. Ganho de peso de leitões alimentados com níveis de babaçu na dieta durante 25 dias.

\section{DISCUSSÃO}

De acordo com Ministério da Agricultura, o farelo de babaçu resultante da extração industrial do óleo pelo uso de um solvente químico deve apresentar os teores máximos de $12 \%$ de umidade, $20 \%$ de proteína bruta, $3 \%$ de gordura e $6 \%$ de cinzas (13).

Para Rostagno et al. (11) o mesocarpo de babaçu apresenta 1,9\% de proteína bruta, $9,7 \%$ de fibra bruta, $0,3 \%$ de extrato etéreo, $2,5 \%$ de matéria mineral, $71,9 \%$ de extrato não nitrogenado, $3.687 \mathrm{kcal} / \mathrm{kg}$ de energia bruta. Contudo, o presente estudo reportou valores de fibra, energia e lipídios superiores aos preconizados pelo Ministério da Agricultura e aos descritos por Rostagno et al. (11), logo, é importante ressaltar que as variações na composição de lipídios possivelmente ocorreram em função do tipo de processamento ao qual foi submetido; a fibra bruta elevada se deve à camada do fruto que foi analisada, pois nos estudos foi empregado apenas o mesocarpo para preparar o farelo, já nesse estudo utilizou-se todo o fruto.

As restrições relacionadas ao uso de ingredientes com elevado teor de fibra bruta são devidas aos componentes da parede celular, visto que não há síntese de enzimas endógenas para atuar na digestão da fração fibrosa dos alimentos de origem vegetal em monogástricos (14). Além disso, elevados níveis de fibra aumentam a motilidade intestinal, reduzindo o tempo de permanência do bolo fecal no trato digestivo, minimizando, assim, a absorção intestinal. Entretanto, não se pode descartar outros aspectos igualmente importantes, por exemplo, o milho apresenta elevada energia metabolizável, possivelmente, pelo nível de fibra, a energia metabolizável para os leitões seja significativamente inferior, assim como a proteína bruta, que no milho é $8,26 \%$, valor $66,10 \%$ superior ao verificado no farelo de babaçu, reduzindo-se a síntese proteica.

Alguns trabalhos com monogástricos demonstram não afetar o crescimento desses animais. Em experimento com tambaquis (Colossoma macropomum), Lopes et al. (15) avaliaram a inclusão de $0 ; 6$ e 12\% de farelo de babaçu na alimentação de peixes tambaqui, sem afetar o crescimento desses animais. Silva Filho (16) avaliaram a inclusão do farelo de 
babaçu em dois diferentes nívies $(0,6$ e $12 \%)$ na dieta de tambaquis juvenis e não conseguiram constatar efeitos sobre o desempenho dos animais.

Para frangos de corte de 21 a 42 dias, níveis de $0 ; 3 ; 6 ; 9$ ou $12 \%$ de farelo de babaçu na dieta, não interferiram no consumo, ganho de peso e conversão alimentar (17). Cruz et al. (18) reportaram efeito similar aos estudos anteriores, com inclusão de $0 ; 6 ; 12$ ou $18 \%$ de farelo de babaçu na alimentação de frangos de corte em fase de crescimento/terminação ( 22 a 42 dias). Entretanto, é relevante esclarecer que nesses estudos, os níveis de inclusão foram abaixo dos testados na presente pesquisa, possivelmente, pelos níveis de fibra na dieta. Assim, os autores podem ter optado por não incluir altos níveis na dieta.

Concordando com os resultados obtidos neste estudo, Gomes et al. (2020) avaliaram a inclusão de farelo de babaçu na dieta de suinos mesticos e identificaram que esse subproduto pode ser utilizado na alimentação destes animais a um nível de até $24 \%$ de farelo de babaçu.

\section{CONCLUSÃO}

Com base nessas informações, foi possível concluir que a adição do farelo de babaçu reduz de forma linear o crescimento de leitões com inclusão de até 30\% na dieta. Assim, recomenda-se sua adição de forma moderada em até $20 \%$.

\section{REFERÊNCIAS}

1. Instituto Brasileiro de Geografia e Estatística - IBGE. Produção da pecuária mundial. Rio de Janeiro: IBGE; 2020.

2. Carneiro MIF, Sakomura NK, Kawauchi IM, Silva EP, Araujo JA, Fernandes JBK, et al. Avaliação do mesocarpo de babaçu (Orbignya ssp) na alimentação de frangos de corte. Ars Vet. 2013;29(3):175-82. doi: http://dx.doi.org/10.15361/2175-0106.2013v29n3p175182.

3. Carneiro BLA, Arévalo-Pinedo A, Scartazzini L, Zuniga ADG, Pinedo RA. Estudo da estabilidade do extrato hidrossolúvel "leite" de babaçu (Orbygnia speciosa) pasteurizado e armazenado sob refrigeração. Rev Bras Frutic. 2014;36(1):232-6. doi: https://doi.org/10.1590/0100-2945-334/13.

4. Araujo JC, Ribeiro NM, Bezerra KCB, Landim LASR. Desenvolvimento de kefir em leite de coco babaçu. Res Soc Dev. 2020;9(11):3559119891. doi: https://doi.org/10.33448/rsd-v9i11.9891.

5. Souza AGC, Sousa NR, Silva SEL. Fruteiras da Amazônia. Brasília: EMBRAPA - SPI; 1996. p. 27-8.

6. Chitarra MIF, Chitarra AB. Pós-colheita de frutas e hortaliças: glossário [Internet]. Lavras: UFLA; 2006 [citado 10 Jun 2020]. Disponível em: https://www.bdpa.cnptia.embrapa.br/consulta/busca? $\mathrm{b}=$ ad\&biblioteca $=$ CPATSA\&busca $=$ autoria:\%22CHITARRA,\%20A.B.\%22

7. Santos FDRP, Santos MGB, Lucena VB, Viana MB, De Castro ADAP. Uso do óleo de coco babaçu (Attalea speciosa) como emoliente em formulação fitocosmética com ação hidratante. Rev Cereus. 2020;12(4):2-13. doi: 10.18605/2175-7275/cereus.v12n4p2-13.

Della Flora MAL, Albuquerque MPF, Mendes M, Rego RS, Viana RR, Cavalcante WM et al. Efeitos da inclusão de diferentes níveis do farelo de babaçu na dieta de suínos. Vet. e Zootec. 2021; v28: 001-008. 
8. Empresa Brasileira de Pesquisa e Agropecuária - EMBRAPA. Babaçu: Programa Nacional de Pesquisa. Brasília: EMBRAPA-DDT; 1984.

9. Tsuji AE, Saito RT, Mori RSF. Automação no agronegócio de pequeno porte: dispositivo para pesagem e empacotamento na cadeia produtiva de morango [trabalho de conclusão de curso]. Curitiba: Universidade Tecnológica Federal do Paraná; 2019.

10. Gomes PEB, Lopes JB, Moreira EMSC, Merval RR, Ribeiro MN, Albuquerque DMN, et al. Farelo de babaçu em dietas para suínos mestiços. Braz J Anim Environ Res. 2020;3(3):1515-24.

11. Rostagno HS, Albino LFT, Donzele JL. Tabelas Brasileiras para aves e suínoscomposição de alimentos e exigências nutricionais. 3a ed. Viçosa: Universidade Federal de Viçosa; 2011.

12. Cunniff P; Association of Official Analytical Chemists - AOAC. Official methods of analysis of AOAC international. 16a ed. Washington, DC: AOAC; 2000.

13. Brasil. Especificações para a padronização, classificação e comercialização interna do óleo, da torta e do farelo de babaçu Orbignia oleifera Burrat. Brasília: Ministério da Agricultura; 1975.

14. Freitas FB, Zanella I, Carvalho AD, Raber MR, Brum Junior BS, Souza JF, et al. Evaluation of multienzyme complex in diets with increasing levels of wheat for layers in the rearing phase. Ars Vet. 2005;21(1):1-6.

15. Lopes JM, Pascoal LAF, Silva Filho FP, Santos IB, Watanabe PH, Araujo DM, et al. Farelo de babaçu em dietas para tambaqui. Rev Bras Saude Prod Anim [Internet]. 2010 [citado 10 Jun 2020];11(2):519-26. Disponível em: https://periodicos.ufba.br/index.php/rbspa/article/viewFile/40365/22468

16. Silva Filho FP. Influência da inclusão do farelo de babaçu nas dietas para juvenis de tambaqui (Colossoma macropomum) sobre: índice hepatossomático, índice de gordura viscero-somática e comprimento relativo do intestino [trabalho de conclusão de curso]. Imperatriz: Faculdade de Imperatriz - FACIMP; 2005.

17. Carneiro APM, Pascoal LAF, Watanabe PH, Santos IB, Lopes JM, Arruda JCB. Farelo de babaçu em rações para frangos de corte na fase final: desempenho, rendimento de carcaça e avaliação econômica. Cienc Anim Bras [Internet]. 2009 [citado 10 Jun 2020];10(1):40-7. Disponível em: https:/www.revistas.ufg.br/vet/article/view/1034/4569

18. Cruz LA, Siqueira, JC, Vaz RGMV, Rodrigues KF, Silva MC, Fonseca FLR, et al. Inclusão da farinha do mesocarpo de babaçu em rações de frangos de corte na fase de crescimento/terminação (22 a 42 dias). Rev Bras Saude Prod Anim. 2015;16(1):223-31. doi: http://dx.doi.org/10.1590/S1519-99402015000100023.

Recebido em: 07/07/2021 Aceito em: 22/11/2021 